\title{
Efecto del modelo y material de construcción de la caja y recubrimiento de los panales de cría en la termorregulación y desarrollo de colonias de Scaptotrigona mexicana
}

Juan Antonio Pérez-Sato ${ }^{a}$

Hugo Rodolfo Salazar-Vargas ${ }^{\text {a }}$

Juan Valente Hidalgo-Contreras ${ }^{a^{*}}$

Natalia Real-Luna ${ }^{\text {a }}$

Héctor Debernardi-De La Vequia ${ }^{\text {a }}$

Roberto De La Rosa-Santamaría ${ }^{\text {a }}$

${ }^{a}$ Colegio de Postgraduados, Campus Córdoba, Km. 348 Carretera Federal CórdobaVeracruz, Congregación Manuel León, 94946, Amatlán de los Reyes, Veracruz, México.

*Autor de correspondencia: e-mail: jvhidalgo@colpos.mx

\section{Resumen:}

La división artificial de colonias de abejas $S$. mexicana es una actividad en la meliponicultura, en donde se reporta la mayor pérdida de colonias. Entre las diversas causas de dicha mortalidad destaca la dificultad de la nueva colonia por mantener la termorregulación de su nido, ya que tradicionalmente se utilizan vasijas de barro en cuyo caso es más difícil mantener una temperatura estable. El objetivo de este estudio fue analizar las interacciones entre modelo de caja, su material de construcción y el recubrimiento de los panales de cría; en la temperatura interna del nido y el desarrollo de colonias obtenidas por división artificial. Se cuantificó el desarrollo de éstas mediante su ganancia de peso final e inicial, y el número de celdas construidas, actividad de la colonia y capacidad del diseño en mantener la temperatura interna del nido. Los resultados muestran que los mejores rangos de temperatura interna se lograron en nidos transferidos a cajas racionales modelo Portugal-Araujo $(P<0.05)$ y Ailton- 
Fontana $(P<0.05)$ cuyos diseños originales fueron modificados al incluir láminas de poliestireno expandible. Además, la temperatura y desarrollo de la colonia se vio favorecida cuando los panales recién transferidos fueron recubiertos con un molde elaborado de cera de abeja Apis mellifera $L$. La interacción positiva entre estos factores permitió proporcionar un rango de temperatura óptimo $\left(27.9\right.$ a $\left.31.0{ }^{\circ} \mathrm{C}\right)$ para el desarrollo de las colonias, las cuales obtuvieron ganancia de peso entre 0.149 a $0.289 \mathrm{~kg}$ y del número de celdas construidas entre 3,511 a 4,956 celdas de cría.

Palabras clave: Termorregulación, Cavidad, Abeja sin aguijón, División artificial, Material aislante, Sustituto de involucro.

Recibido: 10/09/2018

Aceptado: 17/02/2020

\section{Introducción}

La división artificial de colonias es un método utilizado en la meliponicultura y consiste en que a partir de una colonia madre de abejas ${ }^{(1)}$ se obtienen varias colonias hijas a través de los años $^{(2)}$. Posterior a la división la sobrevivencia de la colonia hija llega a ser critica si no se le brindan las condiciones adecuadas ${ }^{(3,4)}$ debido a que las partes del nido son destruidas ${ }^{(5)}$ y la cría de las nuevas colonias queda expuesta a las fluctuaciones de temperatura externa y se estabiliza hasta que las estructuras del nido son nuevamente reconstruidas ${ }^{(6-9)}$. La temperatura óptima del nido oscila entre 31 y $35{ }^{\circ} \mathrm{C}^{(10,11)}$; para conseguirla las obreras cubren el área de cría con pequeñas láminas de cerumen conocido como involucro ${ }^{(12,13)}$ seguido del área de almacenamiento, además las abejas revisten las paredes periféricas con batumen (mezcla de cerumen producido por la abeja y resina de los árboles). Una inadecuada termorregulación del nido puede tener consecuencias graves para la colonia ${ }^{(14)}$, desde un lento crecimiento hasta su muerte ${ }^{(15,16,17)}$. Bajo condiciones de una mala termorregulación del nido, las abejas invierten la mayor parte de sus energías en construir las estructuras de aislamiento, y un menor esfuerzo en recolectar néctar y polen que son esenciales para el desarrollo y sobrevivencia de la colonia ${ }^{(12,18)}$.

Otro factor clave que contribuye en la termorregulación del nido es el tipo de cavidades donde se alojan los nidos de $S$. mexicana ${ }^{(12)}$; las que comúnmente se utilizan de forma tradicional son cavidades naturales como troncos y vasijas de barro. Los troncos poseen paredes gruesas $(>10 \mathrm{~cm})$ que les permiten conservar adecuadamente la temperatura interna del nido ${ }^{(19)}$. Sin embargo, en las cajas racionales existe una mayor fluctuación en la temperatura interna del 
nido debido a que éstas tienen un mayor volumen, altura y menor grosor de las paredes que las cavidades naturales ${ }^{(20)}$.

Debido a la importancia que la termorregulación tiene en el desarrollo de una colonia, es necesario construir cajas racionales que favorezcan el mantenimiento de rangos óptimos de temperatura para el desarrollo de la cría ${ }^{(21)}$. Las cavidades comúnmente usadas para la crianza de $S$. mexicana son a base cajas de madera y vasijas de barro ${ }^{(17,22)}$. Sin embargo, estos materiales no poseen las mejores propiedades térmicas aislantes que ayuden al nido a mantener su temperatura óptima, especialmente en colonias de tamaño pequeño.

En la actualidad se han diseñado modelos de cajas racionales de madera, que facilitan el manejo de la colonia y brindan una mejor termorregulación al nido ${ }^{(6,23,24)}$. El modelo más utilizado para la especie $S$. mexicana es el Portugal-Araujo ${ }^{(23)}$, mientras que el AiltonFontana es utilizado para Tetragonisca angustula y Nannotrigona testaceicornis ${ }^{(24)}$. Este último modelo de caja puede ser modificado para ser usado exitosamente para alojar $S$. mexicana. Ambos modelos pueden ser ampliamente mejorados para brindar una mayor termorregulación al nido de las abejas sin aguijón, si se incluye en su diseño y construcción un material aislante como el poliuretano ${ }^{(6)}$ o poliestireno expandible.

Durante la división artificial de las colonias de abeja sin aguijón, las estructuras del nido esenciales para brindar una termorregulación adecuada al mismo son destruidas durante esta actividad. Esto deja en un estado de vulnerabilidad a las nuevas colonias hijas a los cambios bruscos de temperaturas del ambiente. Una propuesta de contrarrestar esta situación sería diseñar y construir cajas racionales con materiales con propiedades térmicas aislantes y recubrir el área de cría con algún material que cumpla con las funciones del involucro. El objetivo de esta investigación fue analizar en colonias pequeñas obtenidas por división artificial, el efecto que se tiene en la temperatura interna del nido y desarrollo de la colonia de $S$. mexicana; el recubrir sus panales de cría con un material que sustituye al involucro y alojarlos en modelos de cajas racionales construidas con material convencional y materiales con propiedades térmicas aislantes.

\section{Material y métodos}

El estudio se llevó a cabo en el Meliponario ubicado en el área de Permacultura del Colegio de Postgraduados, Campus Córdoba, Amatlán de los Reyes, Veracruz, México, entre los paralelos $18^{\circ} 46^{\prime}$ y $18^{\circ} 58^{\prime} \mathrm{N}$; los meridianos $96^{\circ} 49^{\prime}$ y $96^{\circ} 58^{\prime} \mathrm{O}$; altitud de $600 \mathrm{msnm}$. El clima corresponde a cálido húmedo (88\%) y semicálido húmedo (12\%) con abundantes lluvias en verano, con un rango de temperatura entre 20 a $24{ }^{\circ} \mathrm{C}$ y precipitación de 1,900 a $2,600 \mathrm{~mm}^{(25)}$. El periodo de estudio fue de junio a agosto de 2016 , una vez que se contó con el material experimental, con la construcción del área experimental y buscando evitar el 
período de lluvias, que para el caso de Amatlán de los Reyes es de junio a septiembre; sin embargo, se consideró realizar el experimento en los días donde se presentó un período de días secos y bajo un pabellón para evitar la invasión de la mosquita Pseudohyphocera spp. Otro aspecto por considerar para el período de estudio fue la disponibilidad de alimento para llevar a cabo la división, por lo cual se tiene que realizar cuando se encuentra la época de floración, lo cual al encontrarse en el área de Permacultura las abejas disponen de flora que le proporcionó alimento (néctar y polen) en los meses en los que se realizó la división ${ }^{(3)}$. En este experimento se utilizaron 36 colonias hijas de S. mexicana obtenidas de la siguiente manera: con base en 107 colonias de dos meliponarios del Colegio de Postgraduados, se establecieron 36 colonias madres con un peso promedio de $5 \mathrm{~kg}$ por colonia (rango $=4.52 \mathrm{a}$ $5.86 \mathrm{~kg}$ ). Estas inicialmente estaban alojadas en vasijas de barro, con la finalidad de facilitar su manejo, sólo los panales de cría e involucro con un peso promedio de $265 \mathrm{~g}$ (rango=118 a $384 \mathrm{~g})$ se transfirieron al modelo de caja racional Ailton-Fontana construida de maderapoliestireno. Las colonias recién transferidas se alimentaron con miel de Apis mellifera por 19 días, hasta que las colonias lograron su organización. Después de 84 días, se procedió a la división artificial ${ }^{(26)}$ de las colonias madres para obtener 36 colonias hijas, dando el mismo manejo post transferencia y todas presentaron una reina fecundada hasta los 35 días posterior a la división.

Antes de colocar los panales en la caja, estos fueron pesados en una báscula digital $\left(\right.$ OHAUS $\left.^{\circledR}\right)$, y se registró el peso inicial de panales en cada colonia hija al momento de la división artificial. Este se mantuvo dentro del rango de 0.077 a $0.335 \mathrm{~kg}^{(27)}$. Las nuevas colonias estuvieron compuestas por panales de cría joven y de capullo, abejas obreras y reina fecundada. El diseño del experimento se realizó bajo un diseño en bloques completos al azar (DBCA) con un arreglo factorial completo en los tratamientos (Cuadro 1) y el peso inicial de la colonia como covariable. El arreglo factorial consistió de tres factores $(2 \times 2 \times 3)$, donde el primer factor fue el modelo: modelo Ailton-Fontana (MAF) y modelo Portugal-Araujo (MPA); el segundo factor fue el material utilizado en la construcción de las cavidades racionales: cajas construidas de madera (CCM) y cajas construidas de madera en su exterior, en su parte media contienen una capa de poliestireno expandido y en su parte interna otra capa de madera unidas entre sí formando un emparedado $(\mathrm{CCM}+\mathrm{PE})$; y el tercer factor fue el tipo de material utilizado para recubrir los panales de cría recién transferidos a las cajas con tres niveles: panales de cría transferidos sin recubrimiento (PCTSR) (testigo), panales de cría transferidos recubiertos con cera de Apis mellifera (PCTRCAM) y panales de cría transferidos recubiertos con poliestireno expandible (PCTRPE). La cera de abeja utilizada fue de Apis mellifera $L$. 
Cuadro 1: Arreglo factorial de los tratamientos

\begin{tabular}{lllll}
\hline $\begin{array}{l}\text { Modelo } \\
\text { de caja }\end{array}$ & $\begin{array}{l}\text { Material de } \\
\text { la caja }\end{array}$ & $\begin{array}{l}\text { Material de } \\
\text { panales }\end{array}$ & $\begin{array}{l}\text { Arreglo factorial de los } \\
\text { tratamientos }\end{array}$ & Clave \\
\hline MAF & CCM & PCTSR & MAF-CCM-PCTSR & $\mathrm{T}_{1} *$ \\
MAF & CCM & PCTRCAM & MAF-CCM- PCTRCAM & $\mathrm{T}_{2}$ \\
MAF & CCM & PCTRPE & MAF-CCM-PCTRPE & $\mathrm{T}_{3}$ \\
MAF & CCM+PE & PCTSR & MAF-CCM+PE- PCTSR & $\mathrm{T}_{4}$ \\
MAF & CCM+PE & PCTRCAM & MAF- CCM+PE- PCTRCAM & $\mathrm{T}_{5}$ \\
MAF & CCM+PE & PCTRPE & MAF-CCM+PE- PCTRPE & $\mathrm{T}_{6}$ \\
MPA & CCM & PCTSR & MPA-CCM-PCTSR & $\mathrm{T}_{7} *$ \\
MPA & CCM & PCTRCAM & MPA-CCM- PCTRCAM & $\mathrm{T}_{8}$ \\
MPA & CCM & PCTRPE & MPA-CCM-PCTRPE & $\mathrm{T}_{9}$ \\
MPA & CCM+PE & PCTSR & MPA-CCM+PE- PCTSR & $\mathrm{T}_{10}$ \\
MPA & CCM+PE & PCTRCAM & MPA-CCM+PE- PCTRCAM & $\mathrm{T}_{11}$ \\
MPA & CCM+PE & PCTRPE & MPA-CCM+PE- PCTRPE & $\mathrm{T}_{12}$ \\
\hline
\end{tabular}

$\mathrm{MAF}=$ modelo Ailton-Fontana, $\mathrm{MPA}=$ modelo Portugal-Araujo, $\mathrm{CCM}=$ caja construida de madera, $\mathrm{CCM}+\mathrm{PE}=+$ poliestireno expandible, $\mathrm{PCTSR}=$ panales de cría transferidos sin recubrir, $\mathrm{PCTRCAM}=$ panales de cría transferidos recubiertos con cera de Apis mellifera, PCTRPE= panales de cría transferidos recubiertos con poliestireno expandible. *Tratamientos testigo: T1 para el Modelo Ailton-Fontana (MAF) y T7 para el Modelo Portugal-Araujo (MPA).

Debido a que el peso inicial de las colonias fue muy variable para todas las colonias divididas, se introdujo como covariable al modelo estadístico el peso inicial de las colonias. El modelo estadístico del experimento fue un modelo de efectos mixtos con análisis de covarianza representado de la siguiente manera:

$$
\begin{gathered}
\mathbf{y}_{\mathrm{ijkl}} \mu+\text { bloque }_{1}+\alpha_{\mathrm{i}}+\beta_{\mathrm{j}}+\gamma_{\mathrm{k}}+(\alpha \beta)_{\mathrm{ij}}+(\alpha \gamma)_{\mathrm{ik}}+(\beta \gamma)_{\mathrm{jk}}+(\alpha \beta \gamma)_{\mathrm{ijk}}+\delta\left(\mathrm{x}_{\mathrm{ijkl}}-\overline{\mathrm{x}}_{\mathrm{k}}\right) \\
+\mathrm{e}_{\mathrm{ijkl}} \text { con } \mathrm{i}=1,2 ; \mathrm{j}=1,2 ; \mathrm{k}=1,2,3 \mathrm{yl}=1,2,3
\end{gathered}
$$

Donde:

$\mathbf{y}_{\mathbf{i j k l}}$ es la variable respuesta del i-ésimo efecto del modelo de caja con el j-ésimo tipo de material de construcción y el k-ésimo tipo de recubrimiento del panal de cría en el l-ésimo bloque;

$\boldsymbol{\mu}$ es la media global; bloque ${ }_{1}$ es el efecto aleatorio del l-ésimo bloque con media 0 y varianza $\sigma_{\text {bloque }}^{2}$

$\boldsymbol{\alpha}_{\mathbf{i}}$ es el efecto fijo del i-ésimo del modelo de caja;

$\boldsymbol{\beta}_{\mathbf{j}}$ es el efecto fijo del material de construcción de la caja;

$\gamma_{\mathbf{k}}$ es el efecto fijo del k-ésimo tipo de recubrimiento del panal de cría; $(\boldsymbol{\alpha} \boldsymbol{\beta})_{\mathrm{ij}},(\boldsymbol{\alpha} \boldsymbol{\gamma})_{\mathrm{ik}},(\boldsymbol{\beta} \boldsymbol{\gamma})_{\mathrm{jk}}, \mathbf{y}(\boldsymbol{\alpha} \boldsymbol{\beta} \boldsymbol{\gamma})_{\mathrm{ijk}}$ son las interacciones de los efectos fijos; 
$\boldsymbol{\delta}\left(\mathbf{x}_{\mathbf{i j k l}}-\overline{\mathbf{x}}_{\text {... }}\right)$ es la covariable cuyo coeficiente de regresión lineal es $\delta$ respecto al peso inicial $\mathrm{x}_{\mathrm{ijkl}}$;

$\mathbf{e}_{\mathbf{i j k l}}$ es el error experimental aleatorio el cual se asume independiente e idénticamente distribuido normal con media cero y varianza $\sigma^{2}$.

El análisis de comparación múltiple de las medias se realizó bajo el procedimiento GLIMMIX del paquete estadístico SAS versión 9.4. Las comparaciones de las medías fue a través de la prueba LSD de Fisher.

Las variables respuesta (temperatura) analizadas bajo este diseño se clasificaron de la siguiente manera: temperatura ambiente de día (TAD), temperatura ambiente de noche (TAN) temperatura ambiente promedio (TAP), temperatura interna del nido de día (TIND), temperatura interna del nido de noche (TINN) y temperatura interna promedio (TIP). Respecto al tamaño de la colonia, las mediciones que se realizaron fueron las siguientes: peso inicial de la colonia (PIC), peso final de la colonia (PFC), ganancia de peso de la colonia (GPC), numero de celdas de cría construidas (NCCC), numero de potes totales construidos (NPTC). Finalmente, para observar las relaciones entre las variables respuesta, se realizó un análisis de correlación de Pearson.

Para este estudio se construyeron 18 cajas del MAF con volumen de 5.3 L y 18 cajas MPA con volumen de $4.2 \mathrm{~L}$. En el MAF nueve de dichas cajas fueron construidas completamente de madera (espesor $2.54 \mathrm{~cm}$ ) y nueve de madera (espesor de $2.54 \mathrm{~cm}$ ) y poliestireno expandible. Para el modelo MPA se construyeron bajo las mismas condiciones descritas para MAF.

Por otra parte, los panales de cría transferidos a las 36 cajas construidas con las características mencionadas fueron recubiertos con los siguientes tipos de materiales: con un molde de cera de Apis mellifera (PCTRCAM) (Figura 1) de $12 \mathrm{~cm}$ de diámetro por $6 \mathrm{~cm}$ de alto y un espesor de $0.2 \mathrm{~mm}$ con 4 orificios de ventilación con diámetro de $1 \mathrm{~cm}$ en cada uno de los bordes y con una molde de poliestireno expandible (PCTRPE) (Figura 2) que tuvo características similares a PCTRCAM y también se incluyeron nidos sin este tratamiento como testigo.

Figura 1: Elaboración del recubrimiento para Panales de Cría Transferidos Recubiertos con Cera de Apis mellifera (PCTRCAM)

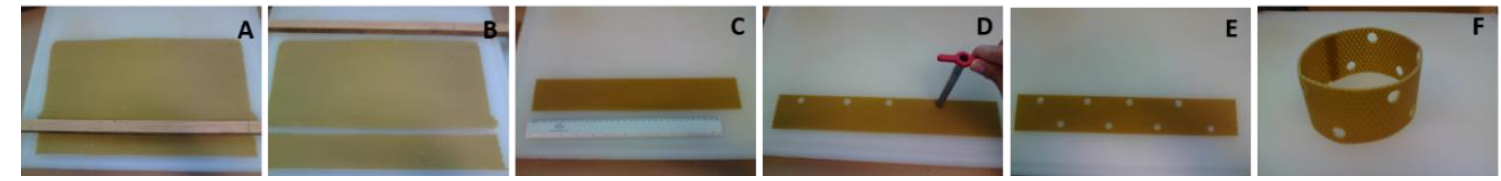

A) Trazo de medidas en lámina de cera estampada de abeja europea Apis mellifera L. B) Corte de láminas. C) Ajuste de medidas $(6 \times 30 \mathrm{~cm})$. D) Elaboración de orificios de ventilación con sacabocados con diámetro de 1 cm. E) Lámina terminada con orificios de ventilación. F) Elaboración de cilindro para colocar dentro la cría recién transferida. Fuente: Elaboración propia. Foto: H.R. Salazar-Vargas. 
Figura 2: Elaboración del recubrimiento para los panales de cría transferidos utilizando un recipiente de poliestireno expandible
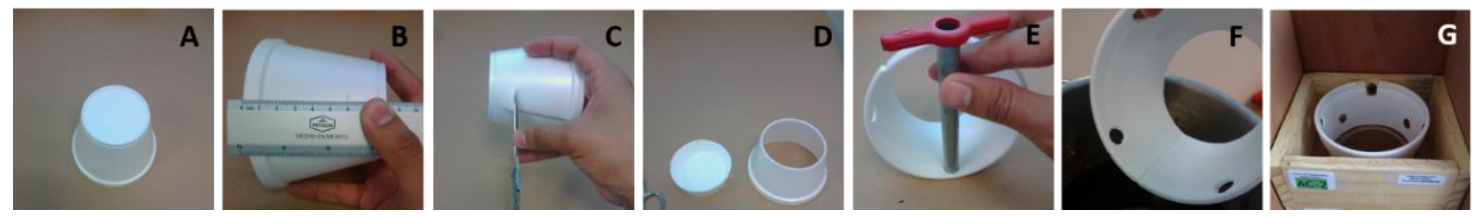

A) Vista del fondo del recipiente de poliestireno expandible B) Trazo de medidas en el recipiente. C) Corte con una tijera de la parte inferior del recipiente. D) La parte inferior del recipiente es eliminado. E)

Elaboración de orificios de ventilación con sacabocados con diámetro de $1 \mathrm{~cm}$. F) Recubrimiento terminado con orificios de ventilación. G) Panales de cría transferidos recubiertos con poliestireno expandible (PCTRPE). Fuente: Elaboración propia. Foto: H.R. Salazar-Vargas.

En el Cuadro 1 se muestran los 12 tratamientos (tres repeticiones cada uno) que resultaron de las combinaciones de tres factores (modelo de caja, material de construcción de las cajas, materiales que recubren el nido) y sus respectivos niveles. Para facilitar la identificación de la combinación se asignó una clave a cada combinación.

Durante 41 días previo al inicio del experimento se hicieron revisiones periódicas donde todas las colonias fueron alimentadas con miel, y polen y se tomaron las medidas de control: limpieza de caja y uso de trampas internas con uso de atrayente (ácido acético al 5\%), en colonias que resultaron positivas a mosca vinagrera (Pseudohypocera spp.). Cuando las colonias presentaron un comportamiento similar, se colocaron en el Meliponario del Área de Permacultura del Campus Córdoba-CP.

Estas fueron distribuidas aleatoriamente en tres bloques de 12 colonias con cada una de las combinaciones, el primer bloque se colocó a $2 \mathrm{~m}$ de distancia del piso, el segundo a $1.5 \mathrm{~m} \mathrm{y}$ el tercero a $1 \mathrm{~m}$ de distancia. Las entradas de los nidos se orientaron hacia el norte. Para evaluar la interacción del modelo de caja, material de construcción de la caja y material que recubre el nido en la temperatura interna, sobre el último disco de cría de cada colonia se colocó un sensor de termómetro digital (VA-DT-1H Avaly ${ }^{\circledR}$ ). Durante 12 semanas en intervalos de cada $4 \mathrm{~h}$, se registró la temperatura interna del nido y la del ambiente. Durante el día se colectaron datos de temperatura a las 0900 h, 1300 h y 1700 h; mientras que durante la noche los horarios fueron $2100 \mathrm{~h}, 0100 \mathrm{~h}$ y $0500 \mathrm{~h}$. Para obtener el incremento de peso de la colonia hija se cuantificó su peso inicial y peso final. Para esto se utilizó una báscula portátil (EQB-100 Torrey ${ }^{\circledR}$ ), además se calculó el número de celdas de cría; esto se realizó de forma indirecta, a través de medir la altura y el diámetro del nido, relacionando el cálculo del número de celdas por centímetro cuadrado mediante un conteo previo para realizar esta estimación, y se contabilizó la cantidad de potes totales visualmente ${ }^{(5)}$. 


\section{Resultados}

Durante el periodo de estudio, el promedio de la temperatura ambiente de día fue de $27.3{ }^{\circ} \mathrm{C}$ $\left(\right.$ rango $=26.7$ a $28.4{ }^{\circ} \mathrm{C}$ ) y la temperatura ambiente de noche de $21.2{ }^{\circ} \mathrm{C} \quad($ rango $=20.8 \mathrm{a}$ $22.4{ }^{\circ} \mathrm{C}$ ). La interacción entre modelo de caja, material de construcción de la caja y material que recubre el nido para la temperatura interna del nido de día (TIND) muestra diferencia significativa $(P<0.05)$, no así para la temperatura interna del nido en la noche (TINN) $(P>0.05)$. Para la TIND entre tratamientos se observa que la media ajustada más alta corresponde a T11 con una media ajustada de $31.5{ }^{\circ} \mathrm{C}$ (Cuadro 2) y la más baja corresponde para T4. Los tratamientos con la media ajustada más alta para la variable TINN fueron T1, T5 y T11 y la más baja fue T4. Entre el tratamiento control T1 y T5 no se observan diferencias significativas $(P>0.05)$. Sin embargo, entre el tratamiento T7 y T11 hubo diferencias significativas $(P<0.05)$.

Cuadro 2: Medias ajustadas $( \pm \mathrm{SD})$ del nido de $S$. mexicana de las variables TIND, TINN y TIP

\begin{tabular}{llll}
\hline \multirow{2}{*}{ Tratamiento } & \multicolumn{4}{l}{ Temperatura interna del nido $\left({ }^{\circ} \mathbf{C}\right)$} \\
\cline { 2 - 4 } & De día (TIND) & De noche (TINN) & Promedio (TIP) \\
\hline $\mathrm{T}_{1}$ & $29.598 \pm 0.715 \mathrm{abc}$ & $27.896 \pm 1.012 \mathrm{a}$ & $28.745 \pm 0.837 \mathrm{ab}$ \\
$\mathrm{T}_{2}$ & $29.391 \pm 0.726 \mathrm{abc}$ & $27.058 \pm 1.03 \mathrm{ab}$ & $28.222 \pm 0.851 \mathrm{ab}$ \\
$\mathrm{T}_{3}$ & $28.361 \pm 0.714 \mathrm{c}$ & $25.386 \pm 1.011 \mathrm{ab}$ & $26.872 \pm 0.836 \mathrm{bc}$ \\
$\mathrm{T}_{4}$ & $25.929 \pm 1.024 \mathrm{~d}$ & $23.395 \pm 1.504 \mathrm{~b}$ & $24.655 \pm 1.223 \mathrm{c}$ \\
$\mathrm{T}_{5}$ & $30.132 \pm 0.69 \mathrm{abc}$ & $27.973 \pm 0.972 \mathrm{a}$ & $29.052 \pm 0.807 \mathrm{ab}$ \\
$\mathrm{T}_{6}$ & $29.497 \pm 0.697 \mathrm{abc}$ & $27.178 \pm 0.984 \mathrm{ab}$ & $28.339 \pm 0.815 \mathrm{ab}$ \\
$\mathrm{T}_{7}$ & $28.736 \pm 0.69 \mathrm{bc}$ & $25.323 \pm 0.971 \mathrm{ab}$ & $27.029 \pm 0.806 \mathrm{bc}$ \\
$\mathrm{T}_{8}$ & $29.771 \pm 0.741 \mathrm{abc}$ & $26.484 \pm 1.054 \mathrm{ab}$ & $28.13 \pm 0.87 \mathrm{ab}$ \\
$\mathrm{T}_{9}$ & $28.811 \pm 0.73 \mathrm{bc}$ & $24.895 \pm 1.037 \mathrm{ab}$ & $26.855 \pm 0.856 \mathrm{bc}$ \\
$\mathrm{T}_{10}$ & $30.292 \pm 0.698 \mathrm{ab}$ & $27.198 \pm 0.985 \mathrm{ab}$ & $28.746 \pm 0.816 \mathrm{ab}$ \\
$\mathrm{T}_{11}$ & $31.358 \pm 0.761 \mathrm{a}$ & $28.137 \pm 1.088 \mathrm{a}$ & $29.75 \pm 0.896 \mathrm{a}$ \\
$\mathrm{T}_{12}$ & $30.148 \pm 0.772 \mathrm{abc}$ & $26.741 \pm 1.106 \mathrm{ab}$ & $28.448 \pm 0.91 \mathrm{ab}$ \\
\hline \multicolumn{5}{c}{${ }^{a b c d}$ Letras diferentes indican diferencia significativa entre tratamientos $(P<0.05)}$.
\end{tabular}

La gráfica de la TIP media ajustada de los tratamientos, durante un periodo de $24 \mathrm{~h}$ (Figura 3) muestra oscilaciones similares entre tratamientos. Las oscilaciones para medias más altas corresponden a T11 y T5 y las mínimas para T4. 
Figura 3: Temperatura media ajustada $( \pm \mathrm{SD})$ de nidos de $S$. mexicana

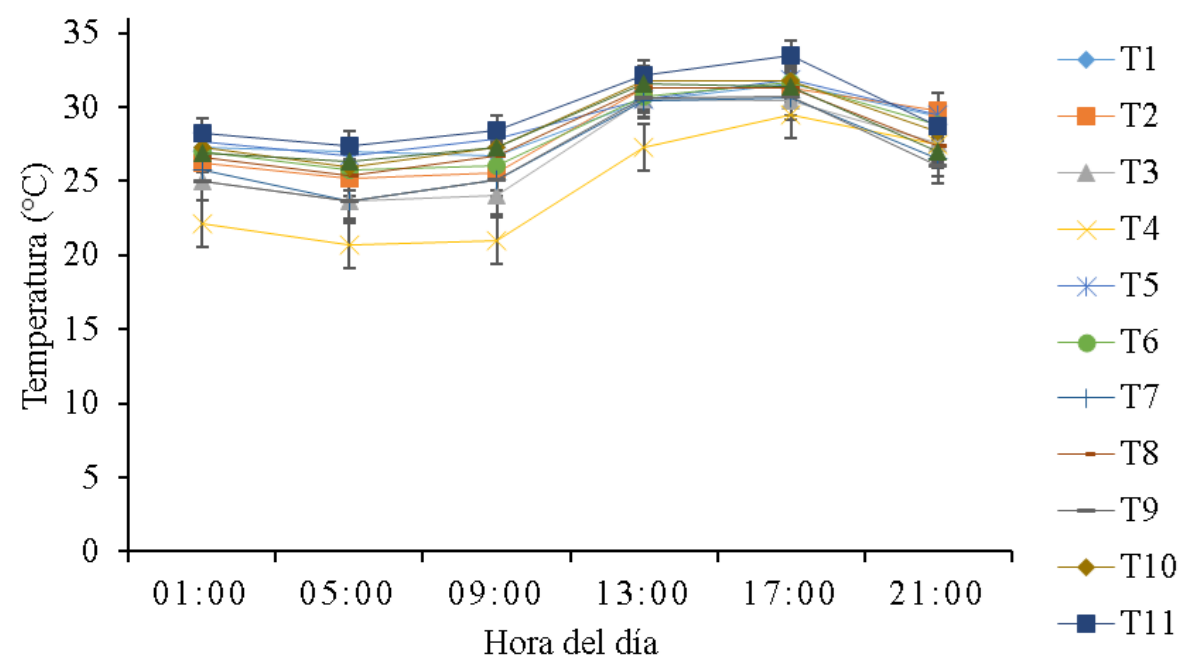

T1(MAF-CCM-PCTSR), T2(MAF-CCM- PCTRCAM), T3(MAF-CCM-PCTRPE), T4(MAF- CCM+PEPCTSR), T5(MAF- CCM+PE- PCTRCAM), T6(MAF-CCM+PE- PCTRPE), T7(MPA-CCM-PCTSR), T8(MPA-CCM- PCTRCAM), T9(MPA-CCM-PCTRPE), T10(MPA-CCM+PE- PCTSR), T11(MPACCM+PE- PCTRCAM) y T12 (MPA-CCM+PE- PCTRPE), durante $24 \mathrm{~h}$.

Los resultados de las medias ajustadas para el peso final de la colonia (PFC), ganancia de peso de la colonia (GPC) se muestran en el Cuadro 3. Así como el número de celdas de cría construidas (NCCC) y número de potes totales construidos (NPTC). Se observaron diferencias significativas para las variables analizadas: PFC $(P=0.0224), \operatorname{GPC}(P=0.0224)$, $\operatorname{NCCC}(P=0.0036)$, no así para NPTC $(P=0.1509)$.

Cuadro 3: Medias ajustadas ( \pm DE) del PIC, PFC y GPC del nido de $S$. mexicana después de 12 semanas del experimento

\begin{tabular}{llll}
\hline \multirow{4}{*}{ Tratamientos } & \multicolumn{3}{l}{ Peso de la colonia $(\mathbf{k g})$} \\
\cline { 2 - 4 } & $\begin{array}{l}\text { Peso inicial } \\
\text { (PIC) }\end{array}$ & $\begin{array}{l}\text { Peso final } \\
\text { (PFC) }\end{array}$ & Ganancia de peso $($ GPC) \\
\hline $\mathrm{T}_{1}$ & $0.2021 \pm 0.0293$ & $0.308 \pm 0.0515 \mathrm{abc}$ & $0.1499 \pm 0.0515 \mathrm{abc}$ \\
$\mathrm{T}_{2}$ & $0.2112 \pm 0.0293$ & $0.294 \pm 0.0524 \mathrm{abc}$ & $0.1358 \pm 0.0524 \mathrm{abc}$ \\
$\mathrm{T}_{3}$ & $0.2015 \pm 0.0293$ & $0.368 \pm 0.0514 \mathrm{ab}$ & $0.2099 \pm 0.0514 \mathrm{ab}$ \\
$\mathrm{T}_{4}$ & $0.3352 \pm 0.0293$ & $0.17 \pm 0.0768 \mathrm{c}$ & $0.0113 \pm 0.0768 \mathrm{c}$ \\
$\mathrm{T}_{5}$ & $0.1653 \pm 0.0293$ & $0.308 \pm 0.0495 \mathrm{abc}$ & $0.1491 \pm 0.0495 \mathrm{abc}$ \\
$\mathrm{T}_{6}$ & $0.1344 \pm 0.0293$ & $0.307 \pm 0.05 \mathrm{abc}$ & $0.1481 \pm 0.05 \mathrm{abc}$ \\
$\mathrm{T}_{7}$ & $0.1594 \pm 0.0293$ & $0.188 \pm 0.0494 \mathrm{c}$ & $0.0295 \pm 0.0494 \mathrm{c}$ \\
$\mathrm{T}_{8}$ & $0.0953 \pm 0.0293$ & $0.233 \pm 0.0537 \mathrm{bc}$ & $0.0748 \pm 0.0537 \mathrm{bc}$ \\
$\mathrm{T}_{9}$ & $0.1026 \pm 0.0293$ & $0.195 \pm 0.0528 \mathrm{c}$ & $0.0369 \pm 0.0528 \mathrm{c}$ \\
$\mathrm{T}_{10}$ & $0.1333 \pm 0.0293$ & $0.254 \pm 0.0501 \mathrm{bc}$ & $0.0953 \pm 0.0501 \mathrm{bc}$ \\
$\mathrm{T}_{11}$ & $0.083 \pm 0.0293$ & $0.448 \pm 0.0554 \mathrm{a}$ & $0.2893 \pm 0.0554 \mathrm{a}$ \\
$\mathrm{T}_{12}$ & $0.077 \pm 0.0293$ & $0.258 \pm 0.0563 \mathrm{bc}$ & $0.0991 \pm 0.0563 \mathrm{bc}$ \\
\hline \multicolumn{4}{l}{}
\end{tabular}


Las comparaciones entre los tratamientos revelaron que para las variables PFC y GPC, T3 (MAF-CCM-PCTRPE) y T11 fueron los más altos (Cuadro 3) y los más bajos T4. Con respecto a las variables NCCC los valores más altos corresponden a T5 y T11 y para NPTC los valores más altos les corresponden a los tratamientos T4, T5 y T11 (Cuadro 4). Existe una correlación significativa entre la TIP y el NCCC $(P<0.0001)$, entre el PIC y NPTC $(P<$ $0.0001)$, y entre el PFC con NCCC $(P<0.0001)$ y NPTC $(P<0.0001)$. El T3 y T11 tuvieron un PFC y GPC mayor en comparación con los otros tratamientos (Figura 3).

Cuadro 4: Medias ajustadas ( \pm SD) del NCCC y NPTC de $S$. mexicana después de 12 semanas del experimento

\begin{tabular}{lll}
\hline Tratamientos & $\begin{array}{l}\text { Numero de celdas de cría } \\
\text { construidas }(\text { NCCC) }\end{array}$ & $\begin{array}{l}\text { Numero de potes totales } \\
\text { construidos }(\text { NPTC) }\end{array}$ \\
\hline $\mathrm{T}_{1}$ & $2372.1 \pm 819.66 \mathrm{bc}$ & $32.801 \pm 8.6603 \mathrm{abc}$ \\
$\mathrm{T}_{2}$ & $3029.8 \pm 829.78 \mathrm{abc}$ & $26.189 \pm 8.7897 \mathrm{bc}$ \\
$\mathrm{T}_{3}$ & $2158.8 \pm 819.01 \mathrm{bcd}$ & $18.558 \pm 8.6521 \mathrm{c}$ \\
$\mathrm{T}_{4}$ & $-496.44 \pm 1110.6 \mathrm{~d}$ & $36.3 \pm 12.286 \mathrm{abc}$ \\
$\mathrm{T}_{5}$ & $3511.5 \pm 797.54 \mathrm{ab}$ & $43.688 \pm 8.3764 \mathrm{ab}$ \\
$\mathrm{T}_{6}$ & $1950.6 \pm 803.82 \mathrm{bcd}$ & $31.724 \pm 8.4572 \mathrm{abc}$ \\
$\mathrm{T}_{7}$ & $1293.7 \pm 796.98 \mathrm{~cd}$ & $21.184 \pm 8.3692 \mathrm{c}$ \\
$\mathrm{T}_{8}$ & $2189.1 \pm 843.39 \mathrm{bcd}$ & $31.932 \pm 8.9632 \mathrm{abc}$ \\
$\mathrm{T}_{9}$ & $1245.2 \pm 833.41 \mathrm{~cd}$ & $25.555 \pm 8.8359 \mathrm{bc}$ \\
$\mathrm{T}_{10}$ & $2739.2 \pm 804.48 \mathrm{bc}$ & $30.551 \pm 8.4657 \mathrm{bc}$ \\
$\mathrm{T}_{11}$ & $4956.8 \pm 862.5 \mathrm{a}$ & $52.006 \pm 9.2057 \mathrm{a}$ \\
$\mathrm{T}_{12}$ & $1784.7 \pm 872.76 \mathrm{bcd}$ & $32.846 \pm 9.3355 \mathrm{abc}$ \\
\hline
\end{tabular}

\section{Discusión}

En este estudio los mejores tratamientos que proporcionan una temperatura interna promedio del nido (TIP), TIND y TINN adecuada para el desarrollo de la cría de $S$. mexicana fueron los tratamientos: T5 (MAF- CCM+PE- PCTRCAM) y T11 (MPA-CCM+PE- PCTRCAM). Esto se explica en parte por las interacciones positivas que resultan de combinar material con propiedades térmicas aislantes (poliestireno expandible) en la construcción de cajas racionales ${ }^{(28)}$, y el recubrir los panales de cría recién transferidos con un material que imita la función que tiene el involucro, como es el caso de la cera de abeja Apis mellifera L. Las interacciones de estas combinaciones impactaron positivamente en la temperatura interna del nido; lo que a su vez influyó en el desarrollo de la colonia mejorando los siguientes aspectos: NCCC, NPTC, PFC y GPC. Como el caso de Melipona subnitida ${ }^{(29)}$ a la cual se le proporcionaron la mayor cantidad de condiciones favorables como temperatura alta y alimento, lo que propició el incremento de celdas de cría. También se ha observado que para 
el caso de Nannotrigona testaceicornis, alojadas en cajas con temperatura artificial durante un periodo invernal, ésta logra mantener la producción de cría y reducir las actividades de termorregulación realizadas por las abejas durante el día o la noche ${ }^{(30)}$.

Comparando los resultados obtenidos en las temperaturas del nido (TIND, TINN, TIP) en los tratamientos T5 $(29.052 \pm 0.807)$ y T11 $(29.75 \pm 0.896)$. Se observa que, aunque no hubo diferencias significativas $(P>0.05)$ entre estos dos tratamientos, en T11 se obtuvieron los mejores resultados con relación a la temperatura interna del nido y desarrollo de la colonia expresado en un mayor número de celdas de cría $(4,956 \pm 862)$ y potes de almacenamiento (52 \pm 9$)$. Estos resultados confirman que la termorregulación interna del nido es importante en los insectos sociales, ya que de ella depende el desarrollo y la supervivencia de la colonia ${ }^{(8,9)}$. El rango de temperatura logrado $\left(28.13\right.$ a $\left.31.35^{\circ} \mathrm{C}\right)$ en este tratamiento se atribuyó a que la caja racional MPA fue diseñada desde un inicio para S. mexicana y posee el volumen adecuado $(4.3 \mathrm{~L})$ para la especie. La interacción entre el volumen interno del MPA, el material con propiedades térmicas aislantes y la protección de la cría utilizando un molde de cera de Apis mellifera L. hacen de este tratamiento, uno de los mejores para la crianza de $S$. mexicana. El rango de temperatura que permite un buen desarrollo de la cría en las abejas nativas es de 31 a $32.3{ }^{\circ} \mathrm{C}^{(11)}$, la temperatura óptima es de $35^{\circ} \mathrm{C}^{(10)}$. El resultado de temperatura del nido obtenido en este tratamiento se encuentra dentro del rango que ha sido calculado como adecuado para el desarrollo esta especie.

El incluir un material con propiedades aislantes (poliestireno expandible) en los modelos de cajas racionales (MAF, MPA) y el recubrir los panales de cría transferidos es vital para proporcionar un rango de temperatura adecuado para el desarrollo de la colonia de tamaño pequeño obtenidas por división artificial. La interacción de estos dos factores juega un papel importante en la termorregulación del nido. Esto se verifica claramente en los resultados obtenidos en los tratamientos T7 $\left(27.029 \pm 0.806{ }^{\circ} \mathrm{C}\right)$ y T11 $\left(29.75 \pm 0.896{ }^{\circ} \mathrm{C}\right)$; en donde el modelo de caja racional MPA fue el mismo para ambos tratamientos. La diferencia radica en que en T7 la caja racional no incluye material con propiedades aislante en su diseño; ni tampoco se recubre la cría con ningún tipo de material. Estas diferencias en los tratamientos originaron diferencias significativas en la temperatura interna del nido $(P<0.05)$. Otras especies de abejas sin aguijón responde de manera similar como es el caso de Melipona colimana que conserva una temperatura homogénea independiente de la temperatura ambiente, sí las condiciones de la cavidad son favorables ${ }^{(31)}$.

En el caso de la caja racional MAF, la cual fue diseñada para las especies $T$. angustula y $N$. testaceicornis $^{(24)}$, fue modificada en sus dimensiones para adaptarla al comportamiento de $S$. mexicana. Su volumen final debido a las modificaciones fue de $5.3 \mathrm{~L}$, ligeramente mayor al MPA. Debido a su volumen mayor, los resultados mostraron que el MAF modificado funciona adecuadamente para alojar S. mexicana, pero se requiere que la cría transferida sea recubierta con un molde de cera de abeja de A. mellifera L. La caja racional MAF modificada 
y completamente de madera también proporcionó una temperatura adecuada al nido cuando los panales de cría fueron recubiertos con cera de abeja.

El tratamiento en donde se registró la TIP del nido más baja fue en el tratamiento T4 (MAFCCM+PE- PCTSR). La TINN fue de $23.395 \pm 1.504$ y tuvo un efecto negativo en el NCCC (-496.44 \pm 1110.6$)$. Un rango de temperatura interna inadecuado provoca que las abejas inviertan la mayor parte de sus energías en construir estructuras de aislamiento, y un menor tiempo en recolectar néctar y polen que son esenciales para el desarrollo y sobrevivencia de la colonia ${ }^{(11,32)}$. En el caso de Scaptotrigona depilis, temperaturas entre 26 a $34^{\circ} \mathrm{C}$, no afectan la sobrevivencia de las crías; sin embargo, temperaturas por debajo de $22{ }^{\circ} \mathrm{C}$ o superiores a $38^{\circ} \mathrm{C}$ causan la mortalidad de la cría, por lo que es muy probable la perdida de las colonias de tamaño pequeño bajo estas condiciones ${ }^{(14)}$.

Es importante señalar que el Modelo Ailton-Fontana (MAF) fue diseñado para T. angustula y $N$. testaceicornis (especies de tamaño pequeño) y aunque en este estudio el modelo fue modificado, adicionando a su diseño original láminas de poliestireno expandible. Sin embargo, esta acción no fue suficiente para proporcionar una temperatura óptima para el desarrollo de colonias de tamaño pequeño de S. mexicana obtenidas por división artificial. Debido al tamaño mayor de volumen $(5.3 \mathrm{~L})$ que tiene el MAF, para el caso de $S$. mexicana se requiere que los panales de cría sean recubiertos con cera de abeja para obtener mejores resultados; tal como se observó en el tratamiento T5 (MAF- CCM+PE- PCTRCAM) en donde se logró una de las mejores TIP del nido.

Las colonias alojadas en el MAF modificado con láminas de poliestireno expandible y sin recubrimientos en los panales, tuvieron los valores más bajos en las siguientes variables: peso final de la cría, ganancia de peso de la cría y número de celdas construidas. Lo anterior se atribuye a la relación que estas variables tienen con la termorregulación del nido. Respecto a la variable cantidad de potes totales, esta fue similar al resto de los tratamientos, esto se explica a que durante el periodo de estudio (primavera-verano), el aprovisionamiento de alimento no se ve afectado debido a que el flujo de néctar es abundante y es favorecido por la temperatura ambiente de día. En otros estudios con Trigona carbonaria se observaron que, para estas colonias, las bajas temperaturas en el nido tuvieron una buena ganancia de peso; debido a la construcción excesiva de involucro o batumen, sin embargo, hubo poco desarrollo de su cría ${ }^{(33)}$.

Los resultados mostraron que la caja racional MAF construida exclusivamente de madera de $3.74 \mathrm{~cm}(2.54 \mathrm{~cm}+1.2 \mathrm{~cm})$ proporciona rangos adecuados de temperatura $(27.896 \mathrm{a}$ $29.598^{\circ} \mathrm{C}$ ) para la cría alojada en su interior. En el caso de M. colimana, el control interno de la temperatura es mejor con un mayor espesor de las paredes en la cavidad ${ }^{(22)}$. La combinación de uso del MAF y el recubrimiento de los panales con el molde de cera de Apis mellifera $L$. mejoran aún más el rango de temperatura interna del nido. 
Las combinaciones de los tratamientos T3, T6, T9 y T12, no se recomiendan debido a que el recubrimiento en los panales de cría con moldes de poliestireno expandible es rechazado por las abejas, dado que al final del período de estudio se observaron mordeduras en este material, en respuesta a que la abeja intentaba manipular para llevar a cabo la termorregulación pasiva del nido.

\section{Conclusiones e implicaciones}

La caja modelo Portugal Araujo (MPA) modificada proporciona un rango de temperaturas adecuadas $\left(25.323-28.736^{\circ} \mathrm{C}\right)$ para el desarrollo de colonias de tamaño pequeño de $S$. mexicana obtenidas por división artificial. Sin embargo, el desarrollo de la colonia y el rango de temperatura mejora ligeramente si los panales de cría son recubiertos con cera de abeja Apis mellifera L. La caja MPA modificada construida con paredes de madera y láminas de poliestireno expandible proporcionaron un mejor rango de temperatura (27.198-30.292 $\left.{ }^{\circ} \mathrm{C}\right)$. La caja modelo Ailton-Fontana (MAF) aunque no fue diseñada para la crianza de $S$. mexicana; mostró que el modelo modificado proporciona rangos adecuados de temperatura (27.896-29.598 ${ }^{\circ} \mathrm{C}$ ) para el desarrollo de esta especie. Dicho rango de temperatura mejoró cuando la caja MAF se modificó adicionando a su diseño láminas de poliestireno expandible y recubriendo los panales de cría con cera de abeja europea (T5). No se recomienda utilizar la caja racional MAF-modificada con poliestireno expandible, sin colocar el recubrimiento de cera de abeja Apis mellifera L. a los panales de cría recién transferidos; debido a que su volumen dificulta que las abejas mantengan un rango de temperatura adecuada para su desarrollo.

\section{Literatura citada:}

1. Cortopassi-Laurino M, Imperatriz-Fonseca VL, Roubik DW, Dollin A, Heard T, Aguilar I, Nogueira-Neto P. Global meliponiculture: challenges and opportunities. Apidologie 2006;37(2): 275-292.

2. Jaffé R, Pope N, Carvalho AT, Maia UM, Blochtein B, Carvalho CAL, ImperatrizFonseca VL. Bees for development: Brazilian survey reveals how to optimize stingless beekeeping. PLoS ONE 2015;10(3).

3. Halcroft MT, Spooner-Hart R, Haigh AM, Heard TA, Dollin A. The Australian stingless bee industry: a follow-up survey, one decade on. J Apic Res 2013;52(2):1-7.

4. Villanueva-Gutiérrez R, Roubik DW, Colli-Ucán W, Güemez-Ricalde FJ, Buchmann SL. A critical view of colony losses in managed Mayan honey-making bees (Apidae: Meliponini) in the heart of Zona Maya. J Kan Ent Soc 2013;86(4):352-362. 
5. Lóriga-Peña W, Álvarez-López D, Fonte-Carballo L, Demedio-Lorenzo J. Población inmadura y reservas de alimentos en colonias naturales de Melipona beecheii Bennett (Apidae: Meliponini) como factores básicos para su salud. Rev Sal Anim 2015;37(1):47-51.

6. Pisté-Mukul MJ. Caracterización y termorregulación del nido de la abeja sin aguijón Scaptotrigona mexicana alojado en cavidades artificiales [tesis maestría]. Campeche, México: Colegio de Postgraduados; 2011.

7. Quezada-Euán JJ. Biología y uso de las abejas sin aguijón de la península de Yucatán, México (Hymenoptera: Meliponini). Edi Univ Aut Yuc 2005;(16).

8. Engels W, Rosenkranz P, Engels E. Thermoregulation in the nest of the Neotropical Stingless bee Scaptotrigona postiça and a hypothesis on the evolution of temperature homeostasis in highly Eusocial bees. Stud Neo F Env 1995;30(4):193-205.

9. Moritz RFA, Southwick EE. Bees as superorganisms - An evolutionary reality. Heidelberg, Germany: Springer; 1992.

10. Márquez-Luna JM. Meliponicultura en México. Dugesiana 1994;1(1):3-12.

11. Roubik DW, Peralta JA. Thermodynamics in nests of two melipona species in Brasil. A Amaz 1983;13(2):453-466.

12. Jones JC, Oldroyd BP. Nest thermoregulation in social insects. Adv Ins Phys 2006;33:153-191.

13. Roubik DW. Stingless bee nesting biology. Apidologie 2006;37(2):124-143.

14. Vollet-Neto A, Menezes C, Imperatriz-Fonseca VL. Behavioural and developmental responses of a stingless bee (Scaptotrigona depilis) to nest overheating. Apidologie 2015;46(4):455-464.

15. Quijano E, González-Acereto J, Quezada-Euán JG. Desarrollo de divisiones de colonias de Melipona beecheii (Hymenoptera, Meliponini) a partir de tres tamaños de población. Bioagrociencias 2008;1(1):4-11.

16. Quezada-Euán JJ. Biología y uso de las abejas sin aguijón de la península de Yucatán, México (Hymenoptera: Meliponini). Edi Univ Aut Yuc 2005;(16).

17. Quezada-Euán JJ, May-Itzá W, González-Acereto JA. Meliponiculture in Mexico: problems and perspective for development. Bee World 2001;82(4):160-167.

18. Biesmeijer JC, Slaa EJ. Information flow and organization of stingless bee foraging. Apidologie 2004;35(2):143-157. 
19. Macias-Macias JO, Quezada-Euan J, Tapia-González JM, Conteras-Escareño F. Nesting sites, nest density and spatial distribution of Melipona colimana Ayala (Hymenoptera: Apidae: Meliponini) in two highland zones of western, Mexico. Sociobiology 2014;61(4): 423-427.

20. Moo-Valle H, Quezada-Euán JJ, Navarro J, Rodríguez-Carvajal LA. Patterns of intranidal temperature fluctuation for Melipona beecheii colonies in natural nesting cavities. J Apic Res 2000;39(2):3-7.

21. Macías-Macías JO, Quezada-Euán JJ, González JM. Effect of lodging type on the internal temperature and humidity of colonies of Melipona colimana (Hymenoptera: Meliponini) from a Mexican temperate zone. J Apic Res 2011;50(3):235-241.

22. Ayala R. Revisión de las abejas sin Aguijón de México (Hymenoptera: Apidae: Meliponini). Fol Ento Mex 1999;106.

23. Guzmán-Díaz M, Balboa C, Vandame R, Albores ML, González-Acereto J. Manejo de las abejas nativas sin aguijón en México. Primer ed: El Colegio de la Frontera Sur; 2011.

24. Barbieri C. Caracterização da meliponicultura e do perfil do meliponicultor no estado de São Paulo: ameaças e estratégias de conservação de abelhas sem ferrão. [tesis maestría]. São Paulo, Brasil: Universidad de São Paulo; 2018.

25. INEGI. Prontuario de información geográfica municipal de los Estados Unidos Mexicanos, Amatlán de los Reyes, Veracruz de Ignacio de la Llave. México; 2009.

26. Kwapong P, Aidoo K, Combey R, Karikari A. Stingless bees, importance, management and utilization. A training manual for stingless beekeeping. First Ed. Accra North, Ghana, Africa: Unimax Macmillan LTD; 2010.

27. Arzaluz A, Obregón F, Jones R. Optimum brood size for artificial propagation of the stingless bee, Scaptoptrigona mexicana. J Apic Res 2002;41(1-2):62-63.

28. Horvath JS. Expanded Polystyrene (EPS) geofoam: An introduction to material behavior. Geo Geomembranes 1994;13(4):263-280.

29. Maia-Silva C, Hrncir M, da Silva CI, Imperatriz-Fonseca VL. Survival strategies of stingless bees (Melipona subnitida) in an unpredictable environment, the Brazilian tropical dry forest. Apidologie 2015;46(5):631-643.

30. Vollet-Neto A, Menezes C, Imperatriz-Fonseca VL. Brood production increases when artificial heating is provided to colonies of stingless bees. J Apic Res 2011;50(3):242247. 
31. Macías-Macías JO, Quezada-Euán JJ, Contreras-Escareño F, Tapia-González JM, MooValle H, Ayala, R. Comparative temperature tolerance in stingless bee species from tropical highlands and lowlands of Mexico and implications for their conservation (Hymenoptera: Apidae: Meliponini). Apidologie 2011;42(6):679-689.

32. Marshall AG. Ecology and natural history of tropical bees. J Trop Eco 1989;9(2):248248.

33. Norgate M, Boyd-Gerny S, Simonov V, Rosa MG, Heard TA, Dyer AG. Ambient temperature influences Australian native stingless bee (Trigona carbonaria) preference for warm nectar. PLOS ONE 2010;5(8). 